\title{
Software para el aprendizaje de operaciones aritméticas a través del ábaco Cranmer para débiles visuals
}

\author{
Software for learning arithmetic operations through the Cranmer abacus for visual \\ weaks
}

SILVA-MARTÍNEZ, Dalia†*, VALVERDE-JARQUÍN, Reyna, GARCÍA-MARTÍNEZ, Ricardo y FLORES-PÉREZ, Mario Andrés

Instituto Tecnológico de Oaxaca / Tecnológico Nacional de México

ID $1^{\text {er }}$ Autor: Dalia, Silva-Martínez / ORC ID: 0000-0002-0561-6459, CVU CONACYT ID: 90232

ID $1^{\text {er }}$ Coautor: Reyna, Valverde-Jarquín / ORC ID: 0000-0002-6505-7804, CVU CONACYT ID: 748083

ID $2^{\text {do }}$ Coautor: Ricardo, García-Martínez / ORC ID: 0000-0001-7277-1478, CVU CONACYT ID: 998270

ID $3^{\text {er }}$ Coautor: Mario Andrés, Flores-Pérez / ORC ID: 0000-0003-2848-6850, CVU CONACYT ID: 988007

DOI: $10.35429 /$ JCA.2019.10.3.1.7

Recibido Abril 30, 2019; Aceptado Junio 30, 2019

\begin{abstract}
Resumen
Este artículo plantea el desarrollo de un software para apoyar a débiles visuales en el aprendizaje del uso del ábaco Cranmer para realizar operaciones matemáticas. Para crear el software se consideró que la persona no puede leer la pantalla, para ello se implementó un lector de pantalla y una navegación a través del scroll y click de tal manera que la persona puede elegir menús y opciones. Se utilizó la metodología de prototipos para ir mejorando cada una de las versiones y adaptándola a las necesidades de los usuarios. El usuario debe contar con un ábaco Cranmer donde representa números, y realiza operaciones matemáticas, posteriormente a través de una cámara el software reconoce los valores representados en el ábaco; el primer nivel es representar números, posteriormente el software plantea una serie de operaciones aritméticas, donde el usuario resuelve y representa en el ábaco y el software evalúa si el usuario realizó la operación correctamente o lo orienta a través de voz para que pueda responder adecuadamente. Existen calculadoras parlantes, pero este software plantea que aprendan a usar el ábaco permitiendo el desarrollo de habilidades matemáticas necesarias en débiles visuales.
\end{abstract}

Abaco Cranmer, Aprendizaje, Débiles visuales

\begin{abstract}
This article proposes a software development to help the sightless people learning arithmetic operations using the Cranmer abacus. To create this software was considered that the sightless person could not read the screen; for that reason, it was implemented a screen reader and navigation through the scroll and click, so the person can choose menus and options. It was used the prototypes methodology to improve each one of the versions and adapt them to the needs of the users. The user has to have a Cranmer abacus where he will represent numbers and do arithmetic operations, later through the use of a camera, the software will recognize the numeric values represented in the abacus. The first level is to represent numbers, later the software proposes a series of arithmetic operations, that the user has to solve and use the abacus to represent the result; the software checks if the answer is correct or gives orientation with a voice to help the user respond accurately. There are talking calculators, but this software proposes the learning with the abacus because it allows to develop mathematic skill needed for the sightless people.
\end{abstract}

Cranmer abacus, Learning, sightless, Blind

Citación: SILVA-MARTÍNEZ, Dalia, VALVERDE-JARQUÍN, Reyna, GARCÍA-MARTÍNEZ, Ricardo y FLORESPÉREZ, Mario Andrés. Software para el aprendizaje de operaciones aritméticas a través del ábaco Cranmer para débiles visuals. Revista de Cómputo Aplicado. 2019, 3-10: 1-7

$\dagger$ Investigador contribuyendo como primer autor. 


\section{Introducción}

El tema de la inclusión ha tomado auge en épocas recientes, el hecho de incorporar a la educación y al trabajo formal a personas que presentan dificultades, se plantea como un reto de la sociedad actual y de autoridades educativas. Las personas que presentan una discapacidad necesitan apoyos para incorporarse al sistema educativo, en otros casos acuden a centros donde reciben atención especial de acuerdo a sus necesidades. Según la Secretaría de Desarrollo Social la discapacidad: "Es la consecuencia de la presencia de una deficiencia o limitación en una persona, que al interactuar con las barreras que le impone el entorno social, pueda impedir su inclusión plena y efectiva en la sociedad, en igualdad de condiciones con los demás" (Sedesol 2016).

Según el Banco Mundial (2011), en el mundo hay aproximadamente 1000 millones de personas que cuentan con una discapacidad y es casi el $15 \%$ de la población, esta cifra es en mayor grado en los países con índice de desarrollo humano bajo.

Aunado a lo anterior, 161 millones de personas tienen problemas de debilidad visual, por lo que es considerada como la segunda causa de discapacidad. Según el INEGI(2010) por cada 100 personas con alguna discapacidad: 55.7 adquirieron esta deficiencia al nacer o por alguna enfermedad, y el $38 \%$ por edad avanzada o quedaron con lesión después de un accidente.

En la encuesta Enadis(2010), uno de cada tres encuestados mencionó que no se respetan los derechos de las personas con discapacidad, por lo mismo su participación en la sociedad se ve limitada y aún cuando se han creado políticas públicas aún falta mucho por hacer.

Una discapacidad aumenta la probabilidad que un niño o adolescente no asista a la escuela o la abandone, y aunque el abandono escolar se da por muchos factores es importante reconocer que no todas las escuelas están preparadas para atender a un estudiante que presenten alguna discapacidad, según la CIF (Clasificación Internacional del funcionamiento de la discapacidad y de la salud) existen muchos tipos de discapacidades, las cuales consideras los siguientes dominios: "funciones y estructuras corporales" y "actividades-participación" (CIF 2001).

\section{Débiles visuales o ciegos}

Según el INEGI (2010) las personas que tienen dificultad para ver se dividen en dos, ciegos: quienes han perdido total o parcialmente la vista, esto puede ser un en un ojo o en ambos; por otro lado los débiles visuales son aquellos que no pueden ver bien, aun usando lentes. Las débiles visuales presentan los dos dominios según CIF, debido a que tienen una deficiencia en la estructura que se presenta por "anomalía" o por "pérdida", las funciones visuales es una de las funciones corporales, pero el dominio anterior provoca también una limitación en actividadesParticipación severa en caso de que no se procure que estas personas puedan ser autosuficientes y tengan acceso a diversos servicios, tal es el caso del sistema educativo.

\begin{tabular}{|c|c|c|c|}
\hline \multicolumn{4}{|c|}{ Clasificación de la discapacidad visual } \\
\hline $\begin{array}{c}\text { Tipos de } \\
\text { discapacidad }\end{array}$ & Profunda & Severa & Moderada \\
\hline Distancia de lectura & $2 \mathrm{~cm}$ & Entre 5 y $8 \mathrm{~cm}$ & Entre $10 \mathrm{y} 15 \mathrm{~cm}$ \\
\hline $\begin{array}{l}\text { Caracteristicas edu- } \\
\text { cacionales }\end{array}$ & $\begin{array}{l}\text { Discapacidad para } \\
\text { realizar tareas vi- } \\
\text { suales gruesas e } \\
\text { imposibilidad para } \\
\text { realizar tareas de } \\
\text { visión de detalle. }\end{array}$ & $\begin{array}{l}\text { Realiza tareas visuales con } \\
\text { inexactitud. Requiere tiempo } \\
\text { para ejecutar una tarea, y } \\
\text { ayudas como lentes olupas o } \\
\text { bien viseras, lentes oscuros, } \\
\text { cuadernos con rayas más } \\
\text { gruesas, plumones para escri- }\end{array}$ & $\begin{array}{l}\text { Efectúa tareas con el } \\
\text { apoyo de lentes e ilu- } \\
\text { minación similares a } \\
\text { los sujetos con visión } \\
\text { normal. }\end{array}$ \\
\hline
\end{tabular}

Tabla 1 Clasificación de debilidad visual

Fuente: Tomada del libro Discapacidad visual: Guía didáctica para la inclusión en educación inicial y básica. (SEP 2010)

\section{La educación para personas con debilidad visual o ceguera.}

En el ámbito educativo uno de los problemas es adaptarse a su entorno, identificando objetos próximos y así evitar accidentes, otro problema es acercar a estas personas a la ciencia, específicamente en el área de matemáticas para que puedan aprender conceptos matemáticos abstractos como el de cantidad numérica, calcular perímetros, describir figuras geométricas, y resolver operaciones matemáticas. En los centros educativos para ciegos, se enseña el uso del ábaco Cranmer para invidentes, pero no todos los estudiantes tienen el mismo razonamiento lógico matemático, lo que dificulta la adquisición de conocimientos y habilidades matemáticas en la Educación Básica: como problemas de adición, sustracción, multiplicación (SEP 2011). 


\section{Software para personas con debilidad visual o ceguera}

Existen pocos desarrollos para personas con debilidad visual o ceguera, por ejemplo JAWS, NonVisual Desktop Access, Orca son lectores de pantalla, es un software parlante que funciona leyendo lo que se muestra en la pantalla, permitiendo a estas personas interactuar con la computadora.

Así como para procesador de textos y envío de archivos, IOS ha lanzado Mobile accessibility para personas invidentes, pero no se encontró alguno que resuelva el problema planteado.

Braille not y blazie para procesador de textos y envío de archivos, IOS a través de este Mobile accessibility para personas invidentes que les permite editar textos.

Tap Tap See fue diseñada para leer etiquetas de productos para que puedan tener un apoyo al momento de realizar compras, es una aplicación de IOS que les permite leer las etiquetas de los productos. Finger Reader es un anillo de audiolectura que permite leer textos, y otro para leer precios de productos en tiendas comerciales.

Nela es un software que enseña a los niños a escribir en braille (el sistema de escritura basado en puntos con relieve) creado en Europa.

En la Guía de Inclusión Digital de alumnos con Discapacidad se encontraron seis software que se recomiendan para este tipo de personas (ver figura 2), el primero que se menciona es un grabador de audio, y el resto son audio cuentos, ninguno es para matemáticas o para el aprendizaje del manejo de la computadora.

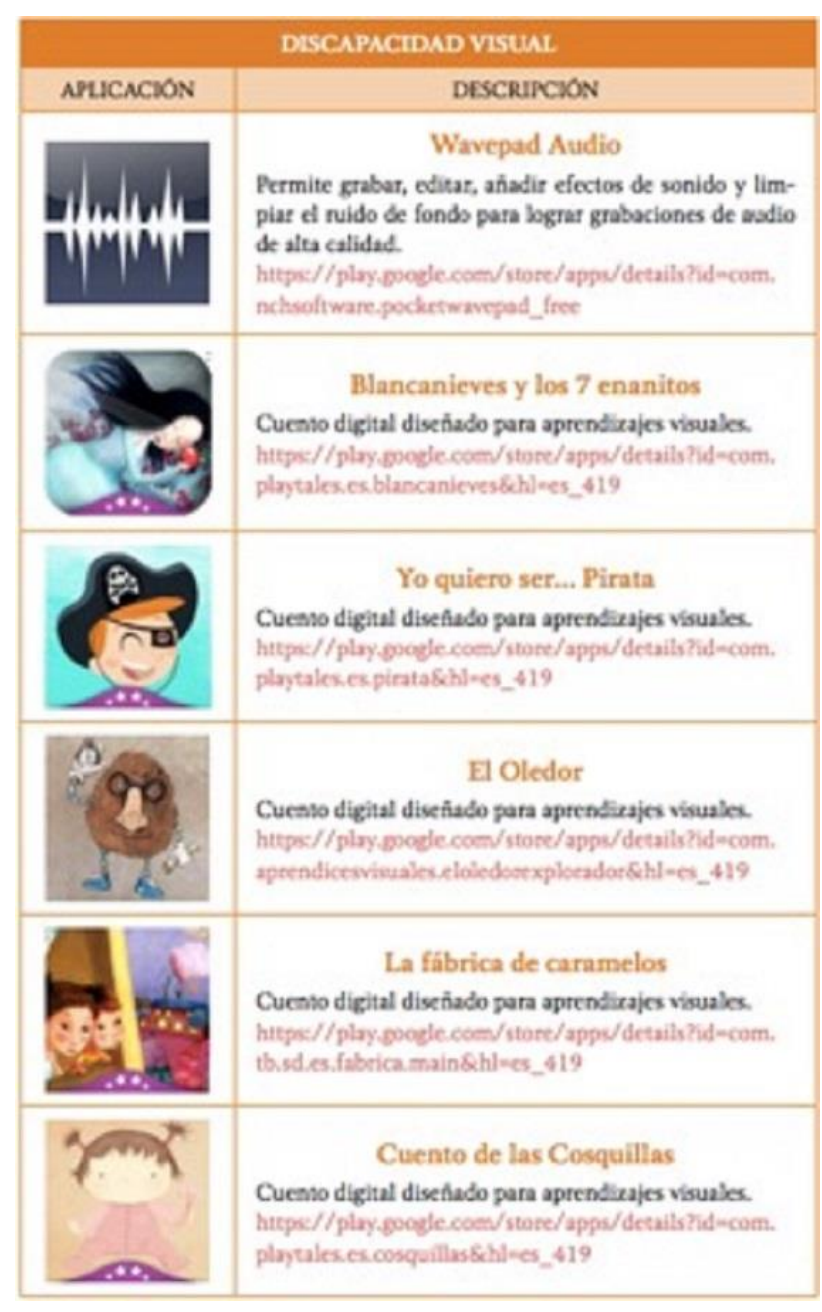

Figura 2 Software para discapacidad visual

Fuente: Tomada del libro Discapacidad Visual: Guía didáctica para la inclusión en educación inicial y básica (SEP 2010)

\section{Metodología a desarrollar}

Como este proyecto es una investigación aplicada, se optó por utilizar el modelo de desarrollo basado en prototipos, ya que ofrece mayor flexibilidad al reutilizar código y la construcción del software es más rápida, pudiendo realizar las pruebas conforme se desarrolla cada parte del proyecto.

Esta metodología consta de seis etapas que son: recolección y refinamiento de requerimientos, diseño rápido, construcción de prototipos, evaluación del prototipo por el cliente, refinamiento del prototipo y producto de ingeniería, estas etapas suelen repetirse mientras los prototipos se van mejorando cada vez. 


\section{Desarrollo del Proyecto}

\section{Recolección y refinamiento de requerimientos}

Los métodos de indagación utilizadas en esta etapa fueron la entrevistas y cuestionarios para conocer los problemas que enfrentan los alumnos en el ámbito escolar(ver figura 3).

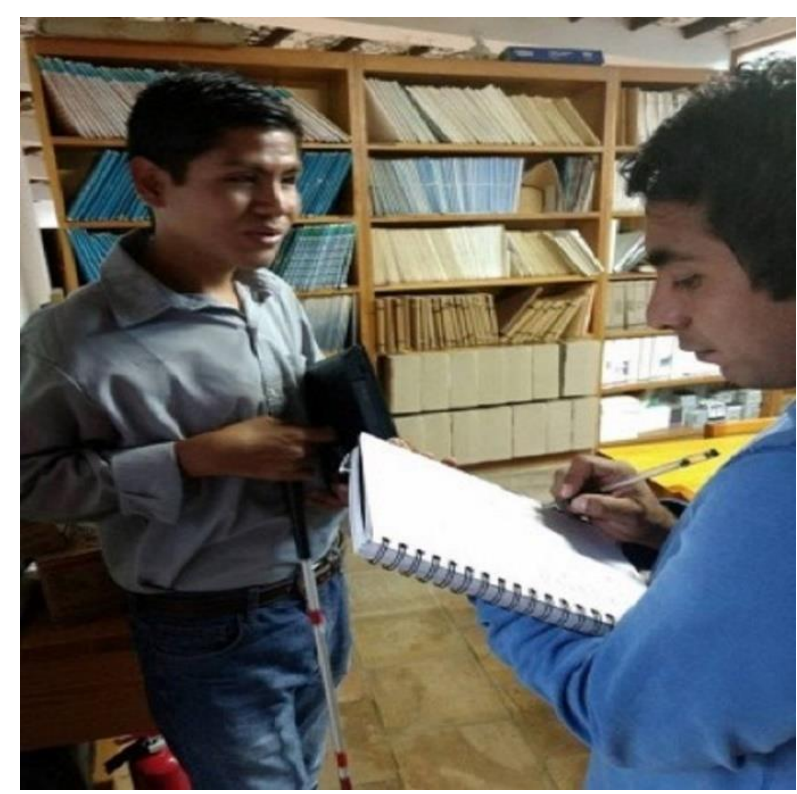

Figura 3 Entrevistando al instructor de ábaco Cranmer, el joven Luis Alberto Reyes

Como resultado de las encuestas se consideró muy importante el desarrollo de un software para facilitar el aprendizaje específicamente en el área de las matemáticas básicas, sobre todo con el uso del Ábaco Cranmer. Se entrevistó a padres de familia que asisten a la biblioteca para ciego Jorge Luis Borges y expresaron que se necesita más material didáctico para invidentes, incluyendo software.

Las personas con debilidad visual o ceguera, que son los usuarios no tenían claro que necesitaban, por ello se optó por la metodología de prototipos. Los requerimientos se determinaron de forma empírica basándose en la observación, y registrando los eventos en una bitácora.

\section{Diseño rápido}

En esta etapa se centró en plasmar los aspectos de software que serán visibles para el usuario, realizando un diseño rápido que cumpla los requisitos planteados por los usuarios; como por ejemplo el diseño de la pantalla principal de la aplicación de matemáticas.

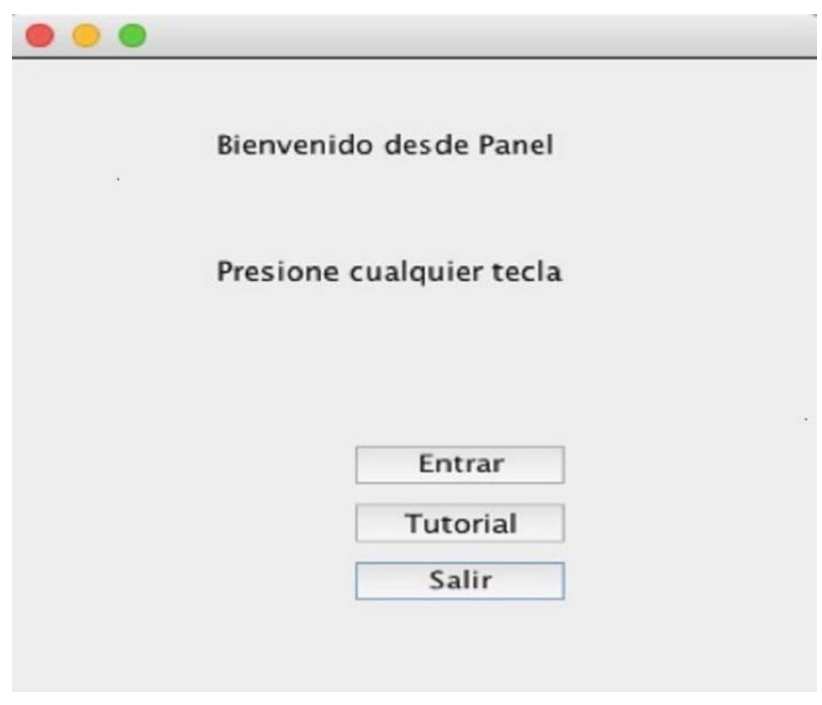

Figura 4 Muestra el diseño de la pantalla principal de la aplicación de matemáticas

\section{Construcción de prototipos}

En cuanto al software para el aprendizaje de las matemáticas, primero se analizó cómo usan los invidentes el ábaco Cranmer para representar valores de números enteros, decimales y fracciones, además del procedimiento para realizar operaciones matemáticas como adición, sustracción y la multiplicación.

Se diseñó el software con el algoritmo de canny, utilizando librerías de Processing para reconocimiento de bordes y pixeles, e identificar la presencia o no de una cuenta en el ábaco Cranmer. La persona coloca el ábaco braille frente a la cámara y este reconoce el número representado. Una vez completado el primer prototipo se procedió a probarlo con los alumnos y Capacitador de la Biblioteca Jorge Luis Borges y personal del DIF.

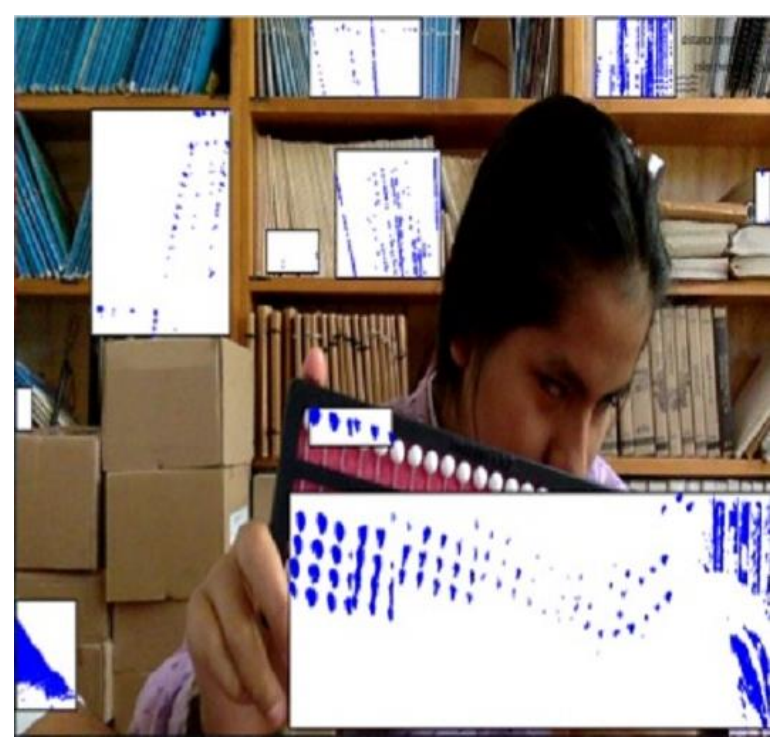

Figura 5 Primera prueba del asistente para braille

SILVA-MARTÍNEZ, Dalia, VALVERDE-JARQUÍN, Reyna, GARCÍA-MARTÍNEZ, Ricardo y FLORES-PÉREZ, Mario Andrés. Software para el aprendizaje de operaciones aritméticas a través del ábaco Cranmer para débiles visuals. Revista de Cómputo Aplicado. 2019. 


\section{Evaluación de prototipo por el cliente}

Se realizó la prueba de usabilidad del software con el ábaco Cranmer. El resultado de ambas pruebas fue de gran utilidad, porque se obtuvieron resultados adecuados para implantación, así como se detectaron problemas a corregir, se obtuvo retroalimentación por parte del usuario (Figura 3). En la prueba del prototipo del ábaco Cranmer, se encontró que el software tiene dificultades con los bordes de color blanco o negro.

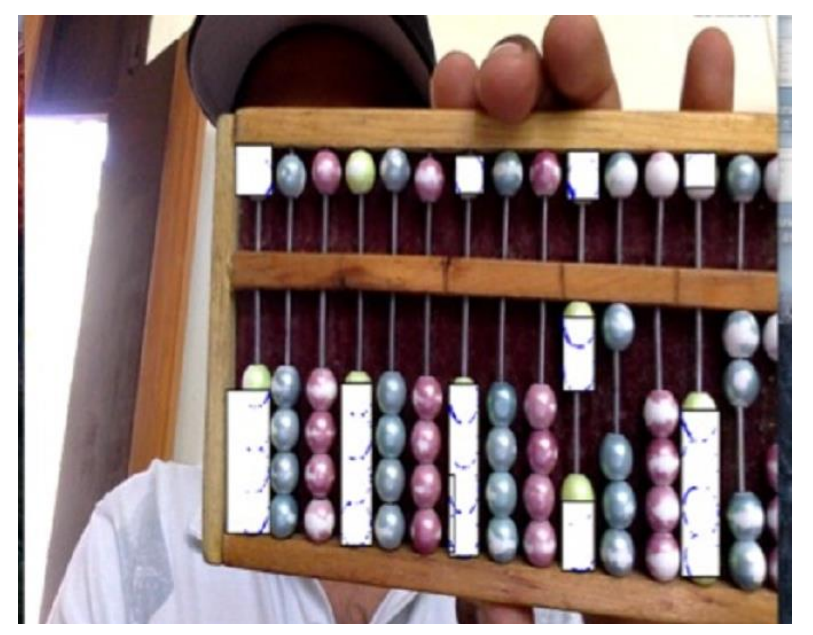

Figura 6 Segunda prueba del asistente para braille. Cuentas de colores

Tercera y última evaluación del prototipo realizada por el cliente para determinar si se requiere algún cambio, también en esta etapa se realizaron entrevistas personales y el seguimiento por parte de los usuarios de la aplicación, de esta manera se obtiene una mejor experiencia del usuario al tener contacto directo con él.

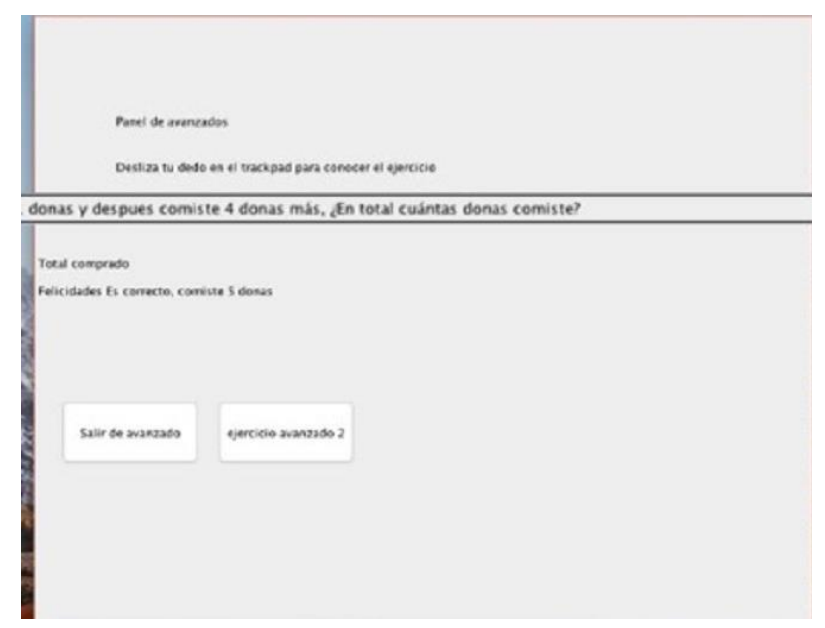

Figura 7 Se observa un ejemplo del uso del abaco. Con la lectura del enunciado
Una vez que el problema es planteado el usuario del software representa el resultado en el ábaco y la cámara de la computadora o dispositivo hace la lectura de las cuentas del ábaco y verifica si la representación es correcta. Con estos ejercicios la persona que es débil visual o ciego puede realizar ejercicios de problemas aritméticos y verificar sus resultados aun cuando no esté el instructor presente.

En la figura 8 hay un ejemplo de cómo funciona esta parte en el software, es importante mencionar que el software funciona con el panel táctil de una computadora.

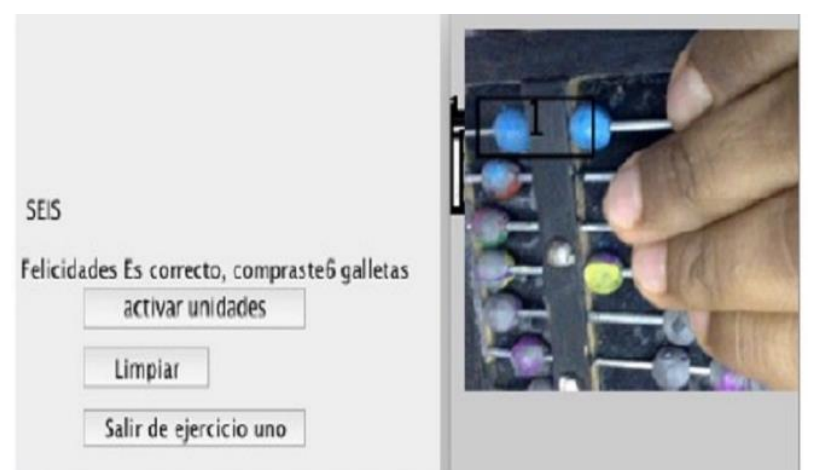

Figura 8 Con base a la figura anterior se presenta el resultado obtenido por parte del usuario

\section{Refinamiento del prototipo}

A partir de los comentarios del cliente y los cambios necesarios en los requisitos, se procedió a construir un nuevo prototipo y así sucesivamente hasta que los requisitos queden totalmente formalizados, y se pueda entonces empezar con el desarrollo del producto final.

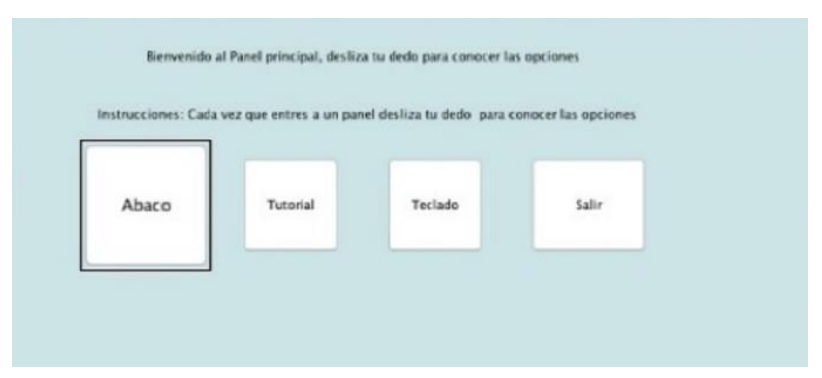

Figura 9 Pantalla principal del contenido del prototipo

Para mejorar el uso del software se implementarán algunos módulos de ayuda, así como el mejoramiento del audio, también se cambió el idioma de los audios. Algunos clientes finales utilizaron el producto e hicieron la recomendación de vincular la aplicación al lector de pantalla propio del sistema operativo. 
Para la entrega final se realizaron pequeños ajustes en cuanto al diseño $y$ corrección en cuanto a la gramática.

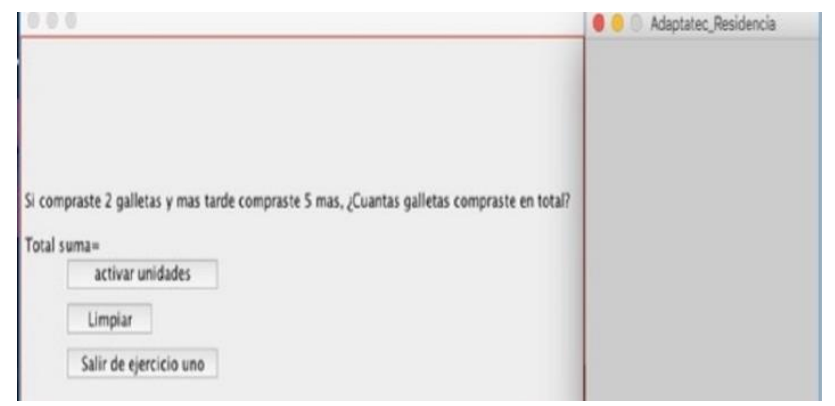

Figura $10 \mathrm{Se}$ realizaron pequeños ajusten en cuanto al diseño

\section{Producto de ingeniería}

Esta etapa es la construcción de la versión final del proyecto y/o prototipo después de haber llevado a cabo cada una de cambios solicitados por el cliente el proyecto se entrega satisfactoriamente.

\section{Resultados}

En la introducción se planteó la búsqueda de información que se realizó para el desarrollo del prototipo, centrándose en la debilidad visual o ceguera como una forma de discapacidad y que presenta dos dominios: el primero corporal y el segundo de actividad participación, esto según la CIF.

También se discutió sobre los desarrollos de software para este tipo de personas, y los que incluye la guía para la inclusión digital, de toda la revisión que se realizó no se encontró alguno para el apoyo del aprendizaje de matemáticas.

En la metodología se mencionó que se usó el desarrollo por prototipos, lo cual fue muy importante para del proyecto, ya que se iban creando versiones mejoradas, y probando, ya que no se tenía bien definido el producto final cuando inició el proyecto.

Con ello se logró que las personas usaran el software de dos maneras: primero para aprender a través de ejercicios como representar un número correctamente en el ábaco Cranmer.
Segundo la computadora le indica que números sumar, la persona lo suma, y posteriormente coloca el ábaco para verificar sus resultados.

\section{Conclusiones}

Con este proyecto se logró diseñar un software para apoyar a personas con debilidad visual o ceguera a que aprendan conceptos matemáticos y a realizar operaciones aritméticas a través del ábaco Cranmer.

Si bien la tecnología cada vez es más accesible a las personas, el uso de Smartphone aumenta cada día, aun es necesario realizar proyectos para que la tecnología también pueda mejorar la calidad de vida de las personas. El desarrollo de software enfocados a este tipo de personas es un área de oportunidad que aún falta mucho por hacer.

\section{Referencias}

CONADIS ( 2010). Encuesta Nacional sobre Discriminación en México. Enadis 2010 https://www.conapred.org.mx/documentos_ced oc/Enadis-PCD-Accss.pdf

INEGI

Discapacidad.

https://www.inegi.org.mx/temas/discapacidad/

INEGI(2010).Cuéntame. http://cuentame.inegi. org.mx/poblacion/discapacidad.aspx?tema=P

OMS (2001). Clasificación Internacional del funcionamiento de la discapacidad y la salud. Organización Mundial de la Salud. Ginebra suiza.

OMS (2011). Informe Mundial de la discapacidad. Disponible en http://www.who.int/disabilities/world_report/20 11/summary_es.pdf

SEDESOL (2016). Diagnóstico sobre la situación de las personas con discapacidad en México. Secretaría de Desarrollo Social https://www.gob.mx/cms/uploads/attachment/fi le/126572/Diagn_stico_sobre_la_Situaci_n_de_ las_Personas_Con_Discapacidad._Mayo_2016. pdf

SEP (2018). Guía de inclusión digital de alumnos con discapacidad. 
SEP (2010). Discapacidad visual Guía didáctica para la inclusión en educación inicial y básica. Recuperado el 11 de 10 de 2017, dehttps://www.gob.mx/cms/uploads/attacment/f ile/106810/discapacidad-isual.pdf

INEGI (2014). La discapacidad en México,datos al 2014. Recuperado el 22 de 10 de 2017, de http://internet.contenidos.inegi.org.mx/contenid os/productos/prod_serv/contenidos/espanol/bvi negi/productos/nueva_estruc/702825090203.pd $\mathrm{f}$ 